Pacific

Journal of

Mathematics

\title{
REGULARITY AT THE BOUNDARY AND TANGENTIAL REGULARITY OF SOLUTIONS OF THE CAUCHY-RIEMANN SYSTEM
}

TRAN Vu KHANH AND GIUSEPPE ZAMPIERI 


\title{
REGULARITY AT THE BOUNDARY AND TANGENTIAL REGULARITY OF SOLUTIONS OF THE CAUCHY-RIEMANN SYSTEM
}

\author{
TRAN Vu KHANH AND GIUSEPPE ZAMPIERI
}

\begin{abstract}
For a pseudoconvex domain $D \subset \mathbb{C}^{n}$, we prove the equivalence of the local hypoellipticity of the system $\left(\bar{\partial}, \bar{\partial}^{*}\right)$ with the system $\left(\bar{\partial}_{b}, \bar{\partial}_{b}^{*}\right)$ induced at the boundary. This develops a former result of ours in which the theory of harmonic extension by Kohn was used. This technique is inadequate for the purpose of the present paper and must be replaced by that of the holomorphic extension.
\end{abstract}

Let $D$ be a pseudoconvex domain of $\mathbb{C}^{n}$ defined by $r<0$ with $C^{\infty}$ boundary $b D$. We use the standard notation $\square=\bar{\partial} \bar{\partial}^{*}+\bar{\partial}^{*} \bar{\partial}$ for the complex Laplacian, $Q(u, u)=$ $\|\bar{\partial} u\|^{2}+\left\|\bar{\partial}^{*} u\right\|^{2}$ for the energy form, and some variants such as $Q_{\mathrm{Op}}(u, u)=$ $\|\mathrm{Op} \bar{\partial} u\|^{2}+\left\|\mathrm{Op} \bar{\partial}^{*} u\right\|^{2}$ for an operator Op. Here $u$ is a $(0, k)$ form belonging to $D_{\bar{\partial}^{*}}$. We similarly define the tangential versions as $\square_{b}, \bar{\partial}_{b}, \bar{\partial}_{b}^{*}$, and $Q_{\mathrm{Op}}^{b}$. We take local coordinates $(x, r)$ in $\mathbb{C}^{n}$, with $x \in \mathbb{R}^{2 n-1}$ being the tangential coordinates and $r$, the equation of $b D$, serving as the last coordinate. We define the tangential $s$-Sobolev norm by $\|u\|_{s}:=\left\|\Lambda^{s} u\right\|_{0}$, where $\Lambda^{s}$ is the standard tangential pseudodifferential operator with symbol $\Lambda_{\xi}^{s}=\left(1+|\xi|^{2}\right)^{s / 2}$. We note that

$$
\left\{\begin{array}{l}
\|\bar{\partial} u\|_{s}^{2}+\left\|\bar{\partial}^{*} u\right\|_{s}^{2}=\sum_{j \leq s} Q_{\Lambda^{s-j} \partial_{r}^{j}}(u, u), \\
\|\bar{\partial} u\|_{s}^{2}+\left\|\bar{\partial}^{*} u\right\|_{s}^{2}=Q_{\Lambda^{s}}(u, u), \\
\left\|\bar{\partial}_{b} u_{b}\right\|_{s}^{2}+\left\|\bar{\partial}_{b}^{*} u_{b}\right\|_{s}^{2}=Q_{\Lambda^{s}}^{b}\left(u_{b}, u_{b}\right) .
\end{array}\right.
$$

We decompose $u$ into a tangential and normal component; that is,

$$
u=u^{\tau}+u^{v},
$$

and further decompose into microlocal components (see [Kohn 2002])

$$
u^{\tau}=u^{\tau+}+u^{\tau-}+u^{\tau 0} .
$$

We similarly decompose $u_{b}$ as $u_{b}^{+}+u_{b}^{-}+u_{b}^{0}$. We use the notation $\bar{L}_{n}$ for the normal $(0,1)$-vector field and $\bar{L}_{1}, \ldots, \bar{L}_{n-1}$ for the tangential ones. Therefore we have the

Keywords: $\bar{\partial}$-Neumann problem, tangential $\bar{\partial}$ system. 
description for the totally real tangential and normal vector fields, denoted by $T$ and $\partial_{r}$ respectively:

$$
\left\{\begin{array}{l}
T=i\left(L_{n}-\bar{L}_{n}\right) \\
\partial_{r}=L_{n}+\bar{L}_{n}
\end{array}\right.
$$

From this, we get back $\bar{L}_{n}=\frac{1}{2}\left(\partial_{r}+i T\right)$. We denote the symbol of a (pseudo)differential operator by $\sigma$ and the partial tangential Fourier transform of $u$ by $\tilde{u}$. We define a holomorphic extension (see [Khanh and Zampieri 2011]) $u^{\tau+(H)}$ of $\left.u^{\tau+}\right|_{b D}$ by

$$
u^{\tau+(H)}=(2 \pi)^{-2 n+1} \int_{\mathbb{R}^{2 n-1}} e^{i x \xi} e^{r \sigma(\dot{T})} \psi^{+}(\xi) \tilde{u}(\xi, 0) d \xi,
$$

where $\dot{T}:=T(x, 0)$. Note that $\sigma(T) \gtrsim\left(1+|\xi|^{2}\right)^{\frac{1}{2}}$ for $\xi$ in supp $\psi^{+}$and $(x, r)$ in a local patch; thus in the integral the exponential is dominated by $e^{-|r|\left(1+|\xi|^{2}\right)^{1 / 2}}$ for $r<0$. Differently from the harmonic extension by Kohn, the present one is well defined only in positive microlocalization. We can think of $u^{\tau+(H)}$ in two different ways: as a modification of $u^{\tau+}$, or as an extension of $u_{b}^{+}$. The property which motivates the terminology of holomorphic extension is

$$
\left\|\bar{L}_{n} u^{\tau+(H)}\right\|=\left\|r \operatorname{Tan} u^{\tau+(H)}\right\| \leq\left\|u_{b}^{\tau+}\right\|_{-\frac{1}{2}} .
$$

This follows from the relationships $\bar{L}_{n}=\frac{1}{2}\left(\partial_{r}+i T\right)$ and $T-\dot{T}=r$ Tan. We have our first relationship between a trace $v_{b}$ and the general extension $v$ ([Kohn 2002] p. 241); for any $\epsilon$ and suitable $c_{\epsilon}$,

$$
\left\|v_{b}\right\|_{s} \lesssim c_{\epsilon}\left\|v\left|\left\|_{s+\frac{1}{2}}+\epsilon\right\| \partial_{r} v\right|\right\|_{s-\frac{1}{2}} .
$$

This is also seen in [Khanh and Zampieri 2011] as the small/large constant argument. As a specific property of our extension we have the reciprocal relation to (1-4):

$$
\left\|r^{k} u^{\tau+(H)}\right\|_{s} \lesssim\left\|u_{b}^{+}\right\|_{s-k-\frac{1}{2}} \cdot
$$

This is readily checked; see [Khanh and Zampieri 2011, (1.12)].

A combination of (1-3) and (1-4) shows that $\bar{L}_{n}$ acts on $u^{\tau+(H)}$ as an operator of order 0 . On the other hand, on the straightening of $b \Omega$ in which $r=x_{n}$, we have that $J \partial_{r}$-i.e., $T$ - coincides with $\partial_{y_{n}}$, and therefore $\bar{L}_{n}$ is the Cauchy-Riemann operator $\partial_{\bar{z}_{n}}$. A reference to the related literature is in order. The extension of generalized functions to half-spaces or wedges of $\mathbb{C}^{n}$ using the decomposition of the $\delta$-function in plane waves as in (1-2) was introduced by Sato, Kashiwara, and Kawai in [Sato et al. 1973] as a general method for microlocal decomposition of the singularities. It has been used, among others, by Boutet de Monvel and Sjöstrand [1976] and by Hsiao [2010] in the study of the singularities of Szegő and Bergman kernels. 
We denote by the symbol $\bar{\partial}^{\tau}$ the extension of the $\bar{\partial}_{b}$ from $b \Omega$ to $\Omega$, which stays tangential to the level surfaces $r \equiv$ const. It acts on tangential forms $u^{\tau}$ and its action is $\bar{\partial}^{\tau} u^{\tau}=\left(\bar{\partial} u^{\tau}\right)^{\tau}$. We denote its adjoint by $\bar{\partial}^{\tau *}$; thus $\bar{\partial}^{\tau *} u^{\tau}=\bar{\partial}^{*}\left(u^{\tau}\right)$. We use the notations $\square^{\tau}$ and $Q^{\tau}$ for the corresponding Laplacian and energy forms. We notice that

$$
Q\left(u^{\tau+(H)}, u^{\tau+(H)}\right)=Q^{\tau}\left(u^{\tau+(H)}, u^{\tau+(H)}\right)+\left\|\bar{L}_{n} u^{\tau+(H)}\right\|_{0}^{2} .
$$

We have to describe how (1-4) and (1-5) are affected by $\bar{\partial}$ and $\bar{\partial}^{*}$.

Proposition 1.1. We have for any extension $v$ of $v_{b}$ that

$$
Q^{b}\left(v_{b}, v_{b}\right) \lesssim Q_{\Lambda^{\frac{1}{2}}}^{\tau}(v, v)+Q_{\partial_{r} \Lambda^{-\frac{1}{2}}}^{\tau}(v, v),
$$

and specifically for $u^{\tau+(H)}$,

$$
Q^{\tau}\left(u^{\tau+(H)}, u^{\tau+(H)}\right) \lesssim Q_{\Lambda^{-\frac{1}{2}}}^{b}\left(u_{b}^{+}, u_{b}^{+}\right)+\left\|u_{b}^{+}\right\|_{-\frac{1}{2}}^{2} .
$$

Proof. We have

$$
\left.\bar{\partial}^{\tau} v\right|_{b D}=\bar{\partial}_{b} v_{b},\left.\quad \bar{\partial}^{\tau *} v\right|_{b D}=\bar{\partial}_{b}^{*} v_{b} .
$$

Then, (1-7) follows from (1-4).

We proceed to prove (1-8). We have $\bar{\partial}^{\tau}=\bar{\partial}_{b}+r$ Tan, and $\bar{\partial}^{\tau *}=\bar{\partial}_{b}^{*}+r$ Tan, which yields

$$
\begin{aligned}
\bar{\partial}^{\tau} u^{\tau+(H)} & =\left(\bar{\partial}_{b} u_{b}\right)^{\tau+(H)}+r \operatorname{Tan} u^{\tau+(H)}, \\
\bar{\partial}^{\tau *} u^{\tau+(H)} & =\left(\bar{\partial}_{b}^{*} u_{b}\right)^{\tau+(H)}+r \operatorname{Tan} u^{\tau+(H)} .
\end{aligned}
$$

Application of (1-5) yields

$$
\begin{aligned}
\left\|\bar{\partial}^{\tau} u^{\tau+(H)}\right\|^{2}+\left\|\bar{\partial}^{\tau *} u^{\tau+(H)}\right\|^{2} & \\
& =\left\|\left(\bar{\partial}_{b} u_{b}\right)^{\tau+(H)}\right\|^{2}+\left\|\left(\bar{\partial}_{b}^{*} u_{b}\right)^{\tau+(H)}\right\|^{2}+\left\|r \operatorname{Tan} u^{\tau+(H)}\right\|^{2} \\
& \lesssim\left\|\bar{\partial}_{b} u_{b}^{+}\right\|_{-\frac{1}{2}}^{2}+\left\|\bar{\partial}_{b}^{*} u_{b}^{+}\right\|_{-\frac{1}{2}}^{2}+\left\|u_{b}^{+}\right\|_{-\frac{1}{2}}^{2}
\end{aligned}
$$

We decompose $u^{\tau+}$ as $u^{\tau+(H)}+u^{\tau+(0)}$, which also serves as a definition of $u^{\tau+(0)}$. Let $\zeta$ and $\zeta^{\prime}$ be cut-offs with $\zeta \prec \zeta^{\prime}$ in the sense that $\left.\zeta^{\prime}\right|_{\operatorname{supp} \zeta} \equiv 1$.

Proposition 1.2. Each of the forms $u^{\#}=u^{v}, u^{\tau-}, u^{\tau 0}, u^{\tau+(0)}, u_{b}^{-}$, and $u_{b}^{0}$ enjoy elliptic estimates; that is,

$$
\left\|\zeta u^{\#}\right\|_{s} \lesssim\left\|\zeta^{\prime} \bar{\partial} u^{\#}\right\|_{s-1}+\left\|\zeta^{\prime} \bar{\partial}^{*} u^{\#}\right\|_{s-1}+\left\|u^{\#}\right\|_{0}, \quad s \geq 2 .
$$

Proof. Estimate (1-10) follows by iteration from

$$
\left\|\zeta u^{\#}\right\|_{s} \lesssim\left\|\zeta \bar{\partial} u^{\#}\right\|_{s-1}+\left\|\zeta \bar{\partial}^{*} u^{\#}\right\|_{s-1}+\left\|\zeta^{\prime} u^{\#}\right\|_{s-1} .
$$


As for $u^{v}$ and $u^{\tau+(0)}$, this latter follows from $\left.u^{v}\right|_{b D} \equiv 0$ and $\left.u^{\tau+(0)}\right|_{b D} \equiv 0$. For the terms with - and 0 , this follows from the fact that $|\sigma(T)| \lesssim|\sigma(\bar{\partial})|$ in the region of 0 -microlocalization, and from $\sigma\left[\bar{\partial}, \bar{\partial}^{*}\right] \leq 0$ and $\sigma(T)<0$ in the negative microlocalization. We refer to (1) in the Main Theorem of [Folland and Kohn 1972] as a general reference, but also give an outline of the proof. We start from

$$
\left\|\zeta u^{\#}\right\|_{1}^{2} \lesssim Q\left(\zeta u^{\#}, \zeta u^{\#}\right)+\left\|\zeta^{\prime} u^{\#}\right\|_{0}^{2}
$$

this is the basic estimate in the case of $u^{v}$ and $u^{\tau+(0)}$ (which vanish at $b D$ ), and it is Lemma 8.6 of [Kohn 2002] for $u^{\tau-}, u^{\tau} 0$ and $u_{b}^{-}, u_{b}^{0}$. Applying (1-12) to $\zeta \Lambda^{s-1} \zeta u^{\#}$ one gets the estimate of tangential norms for any $s$; that is, (1-11) with the usual norm replaced by the triplet norm. Finally, by noncharacteristicity of $\left(\bar{\partial}, \bar{\partial}^{*}\right)$, one passes from tangential to full norms along the guidelines of [Zampieri 2008, Theorem 1.9.7]. The version of this argument for $\square$ can be found in [Kohn 2002, second part of p. 245].

Let $s$ and $l$ be indices.

\section{Theorem 1.3. Consider the estimates}

(1-13) $\left\|\zeta u_{b}\right\|_{s} \lesssim\left\|\zeta^{\prime} \bar{\partial}_{b} u_{b}\right\|_{s+l}+\left\|\zeta^{\prime} \bar{\partial}_{b}^{*} u_{b}\right\|_{s+l}+\left\|u_{b}\right\|_{0} \quad$ for any $u_{b} \in C^{\infty}(b \Omega)$,

(1-14) $\|\zeta u\|_{s} \lesssim\left\|\zeta^{\prime} \bar{\partial} u\right\|_{s+l}+\left\|\zeta^{\prime} \bar{\partial}^{*} u\right\|_{s+l}+\|u\|_{0} \quad$ for any $u \in D_{\bar{\partial}^{*}} \cap C^{\infty}(\bar{\Omega})$,

$(1-15) \quad\|\zeta u\|_{s} \leq \epsilon\left(\|\zeta \bar{\partial} u\|_{s}+\left\|\zeta \bar{\partial}^{*} u\right\|_{s}\right)+c_{\epsilon}\|u\|$

for any $\epsilon$, for suitable $c_{\epsilon}$, and for any $u \in D_{\bar{\partial}^{*}} \cap C^{\infty}(\bar{\Omega})$.

Then (1-13) implies (1-14) and (1-15) implies (1-13) for $l=0$.

Remark 1.4. (i) The above estimates (1-13) and (1-14) for any $s, \zeta, \zeta^{\prime}$ and for suitable $l$, characterize the local hypoellipticity of the system $\left(\bar{\partial}_{b}, \bar{\partial}_{b}^{*}\right)$ and $\left(\bar{\partial}, \bar{\partial}^{*}\right)$ respectively (see [Kohn 2005]). When $l>0$, one says that the system has a loss of $l$ derivatives; when $l<0$, one says that it has a gain of $-l$ derivatives.

(ii) The point in (1-15), as opposed to (1-13) and (1-14), is that we have the same cut-off $\zeta$ in both sides, and also that there is a factor $\epsilon$ of compactness. Though (1-15) is stronger than (1-14), there are wide classes of domains $\Omega$ for which it holds, including all domains of infraexponential type, for which a superlogarithmic estimate holds (see [Baracco et al. 2014]). Indeed, let $R^{s}$ be the pseudodifferential operator defined by $\widetilde{R^{s} u}=\Lambda_{\xi}^{s \sigma(x)} \tilde{u}$ (see [Kohn 2002, p. 234]). On one hand, we have $R^{s} \sim \Lambda^{s}$ modulo operators of order $-\infty$ over $u$ such that $\left.\sigma\right|_{\text {supp } u} \equiv 1$. On the other, we have that $\left[R^{s}, \zeta^{\prime}\right]$ has order $-\infty$ if $\left.\zeta^{\prime}\right|_{\text {supp } \sigma} \equiv 1$ and hence the supports of $\sigma$ and $\dot{\zeta}^{\prime}$ are disjoint. Finally, we have

$$
\left|\zeta^{\prime \prime}\left[\bar{\partial}, R^{s}\right] \zeta^{\prime}\right| \lesssim \log \Lambda R^{s} \zeta^{\prime}
$$


in the sense of operators when $\sigma \prec \zeta^{\prime} \prec \zeta^{\prime \prime}$. Using $R^{s}$ as a substitute for $\Lambda^{s}$, we can prove (1-15) whenever a superlogarithmic estimate holds (see [Kohn 2002, §7]).

Proof. First, it is clearly not restrictive that $u$ and $u_{b}$ have compact support. Because of Proposition 1.2, it suffices to prove (1-13) for $u_{b}^{+}$and (1-14) for $u^{\tau+}$. It is also obvious that we can consider cut-off functions $\zeta$ and $\zeta^{\prime}$ only in tangential coordinates, not in $r$. We start by proving that (1-13) implies (1-14). We recall the decomposition

$$
u^{\tau+}=u^{\tau+(H)}+u^{\tau+(0)}
$$

and begin by estimating $u^{\tau+(H)}$. We then have

$$
\begin{aligned}
\left\|\zeta u^{\tau+(H)}\right\|_{s}^{2} & \underset{(1-5)}{\lesssim}\left\|\zeta u_{b}^{+}\right\|_{s-\frac{1}{2}}^{2} \\
& \underset{(1-13)}{\lesssim} Q_{\Lambda^{s+l-\frac{1}{2} \zeta^{\prime}}}^{b}\left(u_{b}^{+}, u_{b}^{+}\right)+\left\|u_{b}^{+}\right\|_{-\frac{1}{2}}^{2} \\
& \underset{(1-7)}{\lesssim} Q_{\Lambda^{s+l} \zeta^{\prime}}^{\tau}\left(u^{\tau+}, u^{\tau+}\right)+Q_{\partial_{r} \Lambda^{s+l-1} \zeta^{\prime}}^{\tau}\left(u^{\tau+}, u^{\tau+}\right)+\left\|u^{\tau+}\right\|_{0}^{2} .
\end{aligned}
$$

It remains to estimate $u^{\tau+(0)}$. Since $\left.u^{\tau+(0)}\right|_{b D} \equiv 0$, then by 1-elliptic estimates (1-17) $\quad\left\|\zeta u^{\tau+(0)}\right\|_{s}^{2}$

$$
\begin{aligned}
& \underset{(1-11)}{\lesssim} Q_{\Lambda^{s-1} \zeta}\left(u^{\tau+(0)}, u^{\tau+(0)}\right)+\left\|\zeta^{\prime} u^{\tau+(0)}\right\|_{s-1}^{2} \\
& \lesssim Q_{\Lambda^{s-1} \zeta}\left(u^{\tau+}, u^{\tau+}\right)+Q_{\Lambda^{s-1} \zeta}^{\tau}\left(u^{\tau+(H)}, u^{\tau+(H)}\right)+\left\|r \zeta u^{\tau+(H)}\right\|_{s}^{2}+\left\|\zeta^{\prime} u^{\tau+(0)}\right\|_{s-1}^{2} \\
& \lesssim Q_{\Lambda^{s-1} \zeta}\left(u^{\tau+}, u^{\tau+}\right)+\left\|\zeta u^{\tau+(H)}\right\|_{s}^{2}+\left\|\zeta^{\prime} u^{\tau+(H)}\right\|_{s-1}^{2}+\left\|\zeta^{\prime} u^{\tau+(0)}\right\|_{s-1}^{2},
\end{aligned}
$$

where we have used $Q=Q^{\tau}+O(r) \Lambda$ over $h^{\tau+(H)}$; that is, (1-6) in addition to (1-3) in the second inequality, together with the estimate

$$
Q_{\Lambda^{s-1}}^{\tau} \lesssim \Lambda^{s}
$$

in the third. We estimate terms in the last line. First, the term $\left\|\zeta u^{\tau+(H)}\right\| \|_{s}^{2}$ is estimated by means of (1-16). Next, the terms in $(s-1)$-norm can be brought to 0 -norm by combined inductive use of (1-16) and (1-17), and eventually their sum is controlled by $\left\|u^{\tau+}\right\|_{0}^{2}$. We put together (1-16) and (1-17) (with the above further reductions), recall the first part of (1-1) in order to estimate $Q_{\Lambda^{s+l} \zeta^{\prime}}^{\tau}+Q_{\partial_{r} \Lambda^{s+l-1} \zeta^{\prime}}^{\tau}$ in the right side of (1-16), and end up with

$$
\left\|\zeta u^{\tau+}\right\|_{s} \lesssim\left\|\zeta^{\prime} \bar{\partial} u^{\tau+}\right\|_{s+l}+\left\|\zeta^{\prime} \bar{\partial}^{*} u^{\tau+}\right\|_{s+l}+\left\|u^{\tau+}\right\|_{0} .
$$

Finally, by noncharacteristicity of $\left(\bar{\partial}, \bar{\partial}^{*}\right)$, one passes from tangential to full norms in the left side of (1-18) along the guidelines of [Zampieri 2008, Theorem 1.9.7]. The version of this argument for $\square$ can be found in [Kohn 2002] in the second part of p. 245. Thus we get (1-14). 
We prove that (1-15) implies (1-13) for $l=0$. Thanks to $\partial_{r}=\bar{L}_{n}+$ Tan and to (1-3), we have

$$
\partial_{r} u^{\tau+(H)}=\operatorname{Tan} u^{\tau+(H)} \quad \text { and } \quad \bar{L}_{n} u^{\tau+(H)}=r \operatorname{Tan} u^{\tau+(H)} .
$$

It follows that

(1-19) $\left\|\zeta u_{b}^{+}\right\|_{s}^{2}$

$$
\begin{aligned}
& \underset{(1-4)}{\lesssim}\left\|\zeta \zeta u^{\tau+(H)}\right\|_{s+\frac{1}{2}}^{2}+\left\|\partial_{r} \zeta u^{\tau+(H)}\right\|_{s-\frac{1}{2}}^{2} \\
& \lesssim\left\|\zeta u^{\tau+(H)}\right\|_{s+\frac{1}{2}}^{2}+\left\|\bar{L}_{n} \zeta u^{\tau+(H)}\right\|_{s-\frac{1}{2}}^{2} \\
& \underset{(1-15)}{\lesssim} \epsilon\left(Q_{\Lambda^{s+\frac{1}{2} \zeta}}^{\tau}\left(u^{\tau+(H)}, u^{\tau+(H)}\right)+\left\|\zeta \bar{L}_{n} u^{\tau+(H)}\right\|_{s+\frac{1}{2}}^{2}\right) \\
& +c_{\epsilon}\left(\left\|\left|\zeta^{\prime} u^{\tau+(H)}\right|\right\|_{s-\frac{1}{2}}^{2}+\left\|u^{\tau+(H)}\right\|_{0}^{2}\right) \\
& \underset{(1-8)}{\lesssim} \epsilon\left(Q_{\Lambda^{s} \zeta}^{b}\left(u_{b}^{+}, u_{b}^{+}\right)+\left\|\zeta u_{b}^{+}\right\|_{s}^{2}\right)+c_{\epsilon}\left(Q_{\Lambda^{s-1} \zeta^{\prime}}^{b}\left(u_{b}^{+}, u_{b}^{+}\right)+\left\|\zeta^{\prime} u_{b}^{+}\right\|_{s-1}^{2}+\left\|u_{b}^{\tau+}\right\|_{-\frac{1}{2}}^{2}\right) \\
& \lesssim Q_{\Lambda^{s} \zeta^{\prime}}^{b}\left(u_{b}^{+}, u_{b}^{+}\right)+\epsilon\left\|\zeta u_{b}^{+}\right\|_{s}^{2}+c_{\epsilon}\left(\left\|\zeta^{\prime} u_{b}^{+}\right\|_{s-1}^{2}+\left\|u_{b}^{\tau+}\right\|_{-\frac{1}{2}}^{2}\right),
\end{aligned}
$$

where in the second-to-last line we have calculated $\left[\zeta{ }^{(H)}\right]$, which yields

$$
\left\|\zeta u^{\tau+(H)}\right\|\left\|_{s+\frac{1}{2}} \lesssim\right\| \zeta u_{b}^{+}\left\|_{s}+\right\| \zeta^{\prime} u_{b}^{+} \|_{s-1}
$$

(and similarly for $\left[\zeta, Q^{(H)}\right]$ ). We absorb the term with $\epsilon$ and get (1-13).

Since on a pseudoconvex domain the $H^{0}$-ranges of $\square$ and $\square_{b}$ are closed by basic estimates and by [Kohn 1986] respectively, then there are well defined $H^{0}$-inverses denoted by $N$ and $G$, and named the Neumann and Green operators.

Remark 1.5. Equations (1-13) and (1-14) imply local regularity in degree $\geq 2$ of $G$ and $N$ respectively. We first prove regularity for $N$. We start by remarking that

$$
\begin{array}{cc}
\bar{\partial}^{*} N_{q} \text { is regular over } \operatorname{Ker} \bar{\partial} & \text { if } q \geq 2, \\
\bar{\partial} N_{q} \text { is regular over } \operatorname{Ker} \bar{\partial}^{*} & \text { if } q \geq 0 .
\end{array}
$$

In the first case, we set $u=\bar{\partial}^{*} N f$ for $f \in \operatorname{Ker} \bar{\partial}$. We have $\left(\bar{\partial} u=f, \bar{\partial}^{*} u=0\right)$, and hence by (1-14)

$$
\|\zeta u\|_{s} \lesssim\left\|\zeta^{\prime} f\right\|_{s+l}+\|u\|_{0}
$$

To prove the second case, we simply set $u=\bar{\partial} N f$ for $f \in \operatorname{Ker} \bar{\partial}^{*}$ and reason likewise. It follows from (1-20) that the Bergman projection $B_{q}$ is regular in any degree $q \geq 0$. (Notice that even if one started from exact regularity by assuming (1-15), this is perhaps lost by taking the additional $\bar{\partial}$ in $B:=\operatorname{Id}-\bar{\partial}^{*} N \bar{\partial}$.) Finally, 
we exploit formula (5.36) in [Straube 2010] in unweighted norms; that is, for $t=0$ :

$$
\begin{aligned}
N_{q}=B_{q}\left(N_{q} \bar{\partial}\right)\left(\mathrm{Id}-B_{q-1}\right) & \left(\bar{\partial}^{*} N_{q}\right) B_{q} \\
& +\left(\mathrm{Id}-B_{q}\right)\left(\bar{\partial}^{*} N_{q+1}\right) B_{q+1}\left(N_{q+1} \bar{\partial}\right)\left(\operatorname{Id}-B_{q}\right) .
\end{aligned}
$$

Now, in the right side, the $\bar{\partial} N$ 's and $\bar{\partial}^{*} N$ 's are evaluated over Ker $\bar{\partial}^{*}$ and $\operatorname{Ker} \bar{\partial}$ respectively; thus they are regular for $q \geq 2$. The $B$ 's are also regular and therefore such is $N$. This concludes the proof of the regularity of $N$. The proof of the regularity of $G$ is similar, apart from replacing (1-21) by its version for the Green operator $G$ stated in Section 5 of [Khanh 2010].

\section{References}

[Baracco et al. 2014] L. Baracco, T. V. Khanh, and G. Zampieri, "Hypoellipticity of the $\bar{\partial}_{b}$-Neumann problem at exponentially degenerate points", Asian J. Math. (2014), to appear.

[Folland and Kohn 1972] G. B. Folland and J. J. Kohn, The Neumann problem for the CauchyRiemann complex, Annals of Mathematics Studies 75, Princeton University Press, 1972. MR 57 \#1573 Zbl 0247.35093

[Hsiao 2010] C.-Y. Hsiao, Projections in several complex variables, Mém. Soc. Math. Fr. (N.S.) 123, Société Mathématique de France, Paris, 2010. MR 2011m:32004 Zbl 1229.32002

[Khanh 2010] T. V. Khanh, "Global hypoellipticity of the Kohn-Laplacian $\square_{b}$ on pseudoconvex CR manifolds", preprint, 2010. arXiv 1012.5906

[Khanh and Zampieri 2011] T. V. Khanh and G. Zampieri, "Estimates for regularity of the tangential $\bar{\partial}$-system”, Math. Nachr. 284:17-18 (2011), 2212-2224. MR 2859760 Zbl 1229.32013

[Kohn 1986] J. J. Kohn, "The range of the tangential Cauchy-Riemann operator", Duke Math. J. 53:2 (1986), 525-545. MR 87m:32041 Zbl 0609.32015

[Kohn 2002] J. J. Kohn, "Superlogarithmic estimates on pseudoconvex domains and CR manifolds", Ann. of Math. (2) 156:1 (2002), 213-248. MR 2003i:32059 Zbl 1037.32032

[Kohn 2005] J. J. Kohn, "Hypoellipticity and loss of derivatives", Ann. of Math. (2) 162:2 (2005), 943-986. MR 2006k:35036 Zbl 1107.35044

[Boutet de Monvel and Sjöstrand 1976] L. Boutet de Monvel and J. Sjöstrand, "Sur la singularité des noyaux de Bergman et de Szego", pp. 123-164 in Journées: Équations aux Dérivées Partielles de Rennes (Rennes, 1975), edited by J. Camus, Astérisque 34-35, Société Mathématique de France, Paris, 1976. MR 58 \#28684 Zbl 0344.32010

[Sato et al. 1973] M. Sato, T. Kawai, and M. Kashiwara, "Microfunctions and pseudo-differential equations", pp. 265-529 in Hyperfunctions and pseudo-differential equations (Katata, 1971), edited by H. Komatsu, Lecture Notes in Math. 287, Springer, Berlin, 1973. MR 54 \#8747 Zbl 0277.46039

[Straube 2010] E. J. Straube, Lectures on the $\mathscr{L}^{2}$-Sobolev theory of the $\bar{\partial}$-Neumann problem, ESI Lectures in Mathematics and Physics 7, European Mathematical Society (EMS), Zurich, 2010. MR 2011b:35004 Zbl 1247.32003

[Zampieri 2008] G. Zampieri, Complex analysis and CR geometry, University Lecture Series 43, American Mathematical Society, Providence, RI, 2008. MR 2009f:32052 Zbl 1160.32001

Received August 15, 2012. Revised November 26, 2012. 
TRAN VU KHANH

DEPARTMENT OF MATHEMATICS

UNIVERSITY OF PADOVA

Via TRIESTE 63,

I-35121 PADOVA

ITALY

khanh@math.unipd.it

GIUSEPPE ZAMPIERI

DEPARTMENT OF MATHEMATICS

UNIVERSITY OF PADOVA

VIA TRIESTE, 63

I-35121 PADOVA

ITALY

zampieri@math.unipd.it 


\title{
PACIFIC JOURNAL OF MATHEMATICS
}

\author{
msp.org/pjm
}

Founded in 1951 by E. F. Beckenbach (1906-1982) and F. Wolf (1904-1989)

\section{EDITORS}

V. S. Varadarajan (Managing Editor)

Department of Mathematics

University of California

Los Angeles, CA 90095-1555

pacific@math.ucla.edu

Paul Balmer

Department of Mathematics

University of California

Los Angeles, CA 90095-1555

balmer@math.ucla.edu

Daryl Cooper

Department of Mathematics

University of California

Santa Barbara, CA 93106-3080 cooper@math.ucsb.edu

Jiang-Hua $\mathrm{Lu}$

Department of Mathematics

Pokfulam Rd., Hong Kong jhlu@maths.hku.hk
The University of Hong Kong

Don Blasius

Department of Mathematics University of California

Los Angeles, CA 90095-1555

blasius@math.ucla.edu

Robert Finn

Department of Mathematics Stanford University

Stanford, CA 94305-2125

finn@math.stanford.edu

Sorin Popa

Department of Mathematics

University of California

Los Angeles, CA 90095-1555 popa@math.ucla.edu

Paul Yang

Department of Mathematics Princeton University

Princeton NJ 08544-1000

yang@math.princeton.edu

\section{PRODUCTION}

Silvio Levy, Scientific Editor, production@msp.org

\section{SUPPORTING INSTITUTIONS}

ACADEMIA SINICA, TAIPEI

CALIFORNIA INST. OF TECHNOLOGY

INST. DE MATEMÁTICA PURA E APLICADA

KEIO UNIVERSITY

MATH. SCIENCES RESEARCH INSTITUTE

NEW MEXICO STATE UNIV.

OREGON STATE UNIV.

\author{
STANFORD UNIVERSITY \\ UNIV. OF BRITISH COLUMBIA \\ UNIV. OF CALIFORNIA, BERKELEY \\ UNIV. OF CALIFORNIA, DAVIS \\ UNIV. OF CALIFORNIA, LOS ANGELES \\ UNIV. OF CALIFORNIA, RIVERSIDE \\ UNIV. OF CALIFORNIA, SAN DIEGO \\ UNIV. OF CALIF., SANTA BARBARA
}

\author{
Vyjayanthi Chari \\ Department of Mathematics \\ University of California \\ Riverside, CA 92521-0135 \\ chari@math.ucr.edu \\ Kefeng Liu \\ Department of Mathematics \\ University of California \\ Los Angeles, CA 90095-1555 \\ liu@math.ucla.edu \\ Jie Qing \\ Department of Mathematics \\ University of California \\ Santa Cruz, CA 95064 \\ qing@cats.ucsc.edu
}

These supporting institutions contribute to the cost of publication of this Journal, but they are not owners or publishers and have no responsibility for its contents or policies.

See inside back cover or msp.org/pjm for submission instructions.

The subscription price for 2013 is US \$400/year for the electronic version, and \$485/year for print and electronic.

Subscriptions, requests for back issues and changes of subscribers address should be sent to Pacific Journal of Mathematics, P.O. Box 4163, Berkeley, CA 94704-0163, U.S.A. The Pacific Journal of Mathematics is indexed by Mathematical Reviews, Zentralblatt MATH, PASCAL CNRS Index, Referativnyi Zhurnal, Current Mathematical Publications and the Science Citation Index.

The Pacific Journal of Mathematics (ISSN 0030-8730) at the University of California, c/o Department of Mathematics, 798 Evans Hall \#3840, Berkeley, CA 94720-3840, is published twelve times a year. Periodical rate postage paid at Berkeley, CA 94704, and additional mailing offices. POSTMASTER: send address changes to Pacific Journal of Mathematics, P.O. Box 4163, Berkeley, CA 94704-0163.

PJM peer review and production are managed by EditFLOW ${ }^{\circledR}$ from Mathematical Sciences Publishers.

\section{PUBLISHED BY}

mathematical sciences publishers

nonprofit scientific publishing

http://msp.org/

(C) 2013 Mathematical Sciences Publishers 


\section{PACIFIC JOURNAL OF MATHEMATICS}

Volume $265 \quad$ No. $2 \quad$ October 2013

Singularity removability at branch points for Willmore surfaces

YANN BERNARD and TRISTAN RIVIÈRE

On Bach flat warped product Einstein manifolds

QIANG CHEN and CHENXU HE

On plane sextics with double singular points

Alex DeGTyareV

A computational approach to the Kostant-Sekiguchi correspondence

HEIKo DiETRICH and WILLEM A. DE GRAAF

Landau-Toeplitz theorems for slice regular functions over quaternions

GRAZIANO GENTILI and GiUlia SARFATTI

On surgery curves for genus-one slice knots

PATRICK M. GILMER and CHARLES LIVINGSTON

Characterizing abelian varieties by the reduction of the Mordell-Weil group

Chris Hall and Antonella Perucca

Variation of complex structures and the stability of Kähler-Ricci

Solitons

StUART J. HALl and ThOMAS MURPHY

On crossed homomorphisms of the volume preserving diffeomorphism groups

RYOJI KASAGAWA

Regularity at the boundary and tangential regularity of solutions of the Cauchy-Riemann system

TRAN VU KHANH and GIUSEPPE ZAMPIERI

On the Steinberg character of a semisimple $p$-adic group 